\title{
GRISEL-SYNDROM NACH ANTHELIXPLASTIK
}

Florian Durst, Rainer Staudenmaier, Anja C. Pickhard

\section{Einleitung}

Das Grisel-Syndrom beschreibt eine selten vorkommende, nichttraumatische atlantoaxiale Rotations-Subluxation, welche häufig nach Tonsillektomien oder Adenotomien bei Kindern beobachtet werden kann. Wir demonstrieren im Folgenden einen Fall nach einer Anthelixplastik bei einem 9 jährigen Mädchen.

\section{Anamnese \& klinischer Befund}

Anamnese: Die 9-jährige Patientin stellte sich zur Anthelixplastik in unserer Klinik vor. Nach erfolgter Operation mit komplikationsloser oraler Intubation und regelrechtem stationärem Verlauf wurde uns die Patientin am dritten postoperativen Tag mit dem Krankenwagen wegen massivster nuchaler Schmerzen, Schluckunvermögen, Gehunfähigkeit auf Grund starker cervikaler Schmerzen und einem Schiefhals, mit Linksrotation und rechts Lateralflexion des Kopfes und Myogelosen im Bereich der M. sternocleidomastoideus der rechten Seite eingeliefert. Schmerzen im Bereich des Operationsgebietes wurden von der Patientin verneint.

Klinischer Befund: Es zeigten sich reizlose Wundverhältnisse sowie ein kosmetisch sehr befriedigendes Ergebnis. Der Hals-Nasen-OhrenBefund stellte sich altersentsprechend unauffällig dar. Es zeigten sich insbesondere keine Schleimhautläsionen im Naso- oder Oropharynx, die eine fortgeleitete Entzündung nahelegten. Aus orthopädischer Sicht bestand ein massiv schmerzhaft rigider Schiefhals mit Linksrotation und rechts Lateralflexion des Kopfes ohne peripheres sensomotorisches Defizit.

Fig. 1
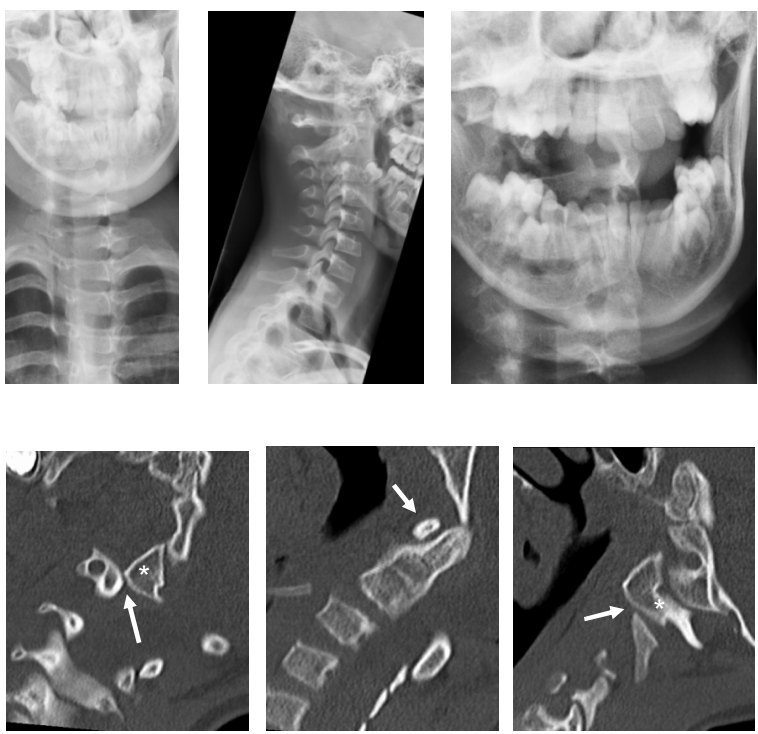

\section{Diagnostik}

Laborchemie. In der von uns durchgeführten laborchemischen Untersuchung fanden sich keinerlei Entzündungszeichen (CRP 0,3 $\mathrm{mg} / \mathrm{dl}$, Leukozyten 6,24 G/l).

Röntgen der Halswirbelsäule in 2 Ebenen, Dens-Spezialaufnahme. Die Dens-Aufnahme war bei Fehlrotation und Überlagerung nicht beurteilbar und auch die a.p. Aufnahme war deutlich fehlrotiert (Fig. 1).

CT der HWS. Es zeigte sich eine Rotations-Subluxation der Articulatio atlantoaxialis lateralis beidseits mit Rotation des Atlas auf dem Axis nach links. Außerdem fand sich eine Subluxationsstellung der rechten Hinterhauptskondyle im rechten Atlantooccipitalgelenk nach dorsal. Eine Fraktur konnte ausgeschlossen werden (Fig. 2).

\section{Therapie \& Verlauf}

Die Patientin wurde seit der Operation prophylaktisch mit Clindamycin therapiert. Bei Aufnahme erhielt sie zudem Antiphlogistika (Ibuprofen), Analgetika (Metamizol) und Tetrazepam (Musaril) zur Muskelrelaxation sowie Wärmebehandlungen für 7 Tage. Nachdem sich keine deutliche Besserung der Beschwerdesymptomatik zeigte, erfolgte die bildgebende Diagnostik, bei der sich eine Subluxation im atlantoaxialen Gelenk zeigte. Daraufhin wurde eine Reposition in Intubationsnarkose durchgeführt. Im Anschluss daran wurde die Patientin für 2 Wochen mit einer Minerva-Halsorthese zur Ruhigstellung der HWS versorgt. In den durchgeführten Nachsorgeuntersuchungen war die Patientin schmerzfrei und es zeigte sich eine achsengerechte Kopf-Hals-Stellung (Fig. 3).
Fig. 3
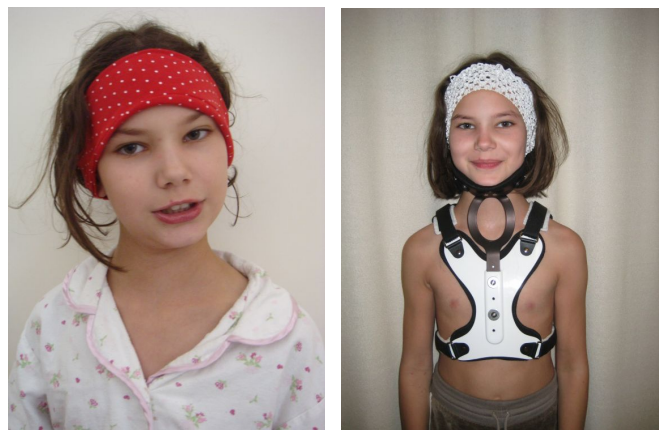

Fig. 1

(a)

Fig. 2

MSCT, Knochenfenster, sagittale Schichtführung:

Ansicht linke Seite: Subluxationsstellung der linken Massa lateralis des Atlas (Stern) gegenüber der linken Massa lateralis des Axis nach dorsal (Pfeil)

Ansicht Mitte: Regelrechte Stellung zwischen vorderem Atlasbogen (Pfeil) und Dens axis Ansicht rechte Seite: Subluxationsstellung der rechten Massa lateralis des Atlas (Stern) gegenüber der rechten Massa lateralis des Axis nach ventral (Pfeil)

Fig. 3

Patientin vor und nach Reposition

\section{Fazit}

Der sehr häufig beobachtete Schiefhals bei Kindern nach HNO Eingriffen auf Grund von Muskelverspannung oder Wundschmerz sollte be therapierefaktärer Persistenz weiter diagnostisch abgeklärt werden. Falls sich eine atlanto-axiale Subluxation diagnostizieren lässt, sollte angelehnt an die Fielding-Klassifikation eine stadiengerechte Therapie erfolgen, Die Prognose bei frühzeitiger Diagnosestellung und rascher Therapie ist sehr gut. Bei verspäteter oder falscher (konservativ physiotherapeutischer) Therapie kann es zu neurologischen Schäden bis hin zur Querschnittssymptomatik kommen. 